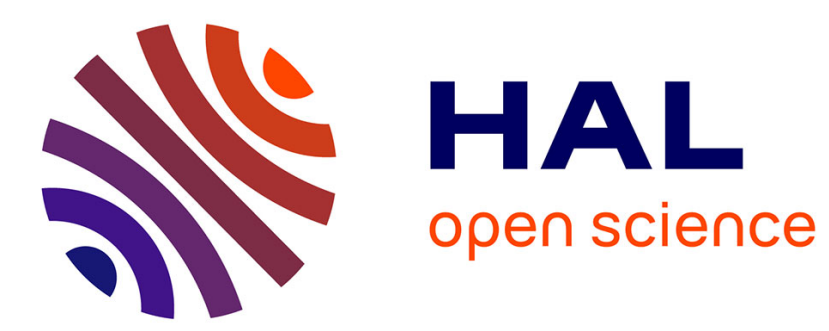

\title{
SEMI-CLASSICAL CALCULATION OF THE SPIN-ISOSPIN RESPONSE FUNCTIONS
}

\author{
G. Chanfray
}

\section{To cite this version:}

G. Chanfray. SEMI-CLASSICAL CALCULATION OF THE SPIN-ISOSPIN RESPONSE FUNCTIONS. International Workshop On Semiclassical And Phase Space Approaches To The Dynamics Of The Nucleus, 1987, Aussois, France. pp.C2-65-C2-69, 10.1051/jphyscol:1987210 . jpa-00226475

\section{HAL Id: jpa-00226475 https://hal.science/jpa-00226475}

Submitted on 1 Jan 1987

HAL is a multi-disciplinary open access archive for the deposit and dissemination of scientific research documents, whether they are published or not. The documents may come from teaching and research institutions in France or abroad, or from public or private research centers.
L'archive ouverte pluridisciplinaire HAL, est destinée au dépôt et à la diffusion de documents scientifiques de niveau recherche, publiés ou non, émanant des établissements d'enseignement et de recherche français ou étrangers, des laboratoires publics ou privés. 


\title{
SEMI-CLASSICAL CALCULATION OF THE SPIN-ISOSPIN RESPONSE FUNCTIONS
}

\author{
G. CHANFRAY \\ Institut de physique Nucléaire (and IN2P3), Université \\ Claude Bernard Lyon-1, 43, bd du 11 novembre 1918. \\ F-69622 Villeurbanne Cedex, France
}

\begin{abstract}
RESUME
Nous présentons une approche semi-classique qui va au-delà de l'approximation de Thomas-Fermi pour le calcul des réponses nucléaires. Notre formalisme est appliqué aux réponses de spin-isospin. Lorsque celles-ci sont sondées par des hadrons, nous montrons que les corrections surfaciques en $\hbar^{2}$ conduisent $\grave{a}$ une très nette diminution du rapport longitudinal/transuerse.
\end{abstract}

\begin{abstract}
We present a semi-classical calculation of the nuclear response functions beyond the Thomas-Fermi approximation. We apply our formalism to the spin-isospin responses and show that the surface peaked $h^{2}$ corrections considerably decrease the ratio longitudinal/transuerse as obtained through hadronic probes.
\end{abstract}

The quasi-elastic nuclear responses at intermediate momentum transfer $q \sim$ 2-3 $m_{\pi}$ are mainly sensitive to average volume properties. Consequently we expect that a phase "space approach, as Thomas-Fermi (TF) theory, will provide a correct description of the excitation mechanism in the continuum. This feature has been explicitely proven in the case of the single particle response for which the TF result compares extremely well to its quantum mechanical analog [1]. Moreover, a realistic TF-RPA calculation [2, 3] gives a satisfactory description of both longitudinal and transverse structure functions measured in (e,e') scattering experiments [4].

When applied to the problematic of spin-isospin responses, the TF theory [3], as the nuclear matter approach [5], predicts marked collective effects involving the whole nuclear interior. The transverse response $R_{T}$ should be quenched even at momenta of order $2 \mathrm{Fm}^{-1}$ since the short range repulsion (usually characterized by a Landau-Migdal parameter $g^{\prime}$ ) still dominates over the attractive tho exchange. The experimental response [4], known from $\left(e, e^{\prime}\right)$ data is compatible with this collective behaviour. On the other hand, the longitudinal response $R_{1}$, sensitive to the long-range pion exchange potential, should exhibit collective effects of opposite nature as a reflect of the nuclear pionic field enhancement [5]. Thus a net contrast between $R_{L}$ and $R_{T}$ is expected at momentum transfer around $2 \mathrm{Fm}^{-1}$.

These theoretical ideas have strongly motivated a $\left(\vec{p}, p^{\vec{t}}\right)$ experiment which has been performed in Los alamos [6]. The resulting data were first rather deceiving as they showed no contrast and seemed to contradict the existence of the contrast. We will show that those data do not actually rule out the expected collective nature of the spin-isospin responses. The reason lies in the fact that protons essentially probe the nuclear surface where specific effects depending on the gradient of the density show up. Those effects, which cannot be simulated by simply lowering the density in the TF scheme, will be incorporated through a Wigner-Kirkwood expansion of the response up to order $\pi^{2}$. The surface peaked $\hbar^{2}$ correction terms acquire an important weight when the nucleus is probed by hadrons and then strongly mask the collective reshaping of the T.F (i.e $\hbar^{\circ}$ ) response. 


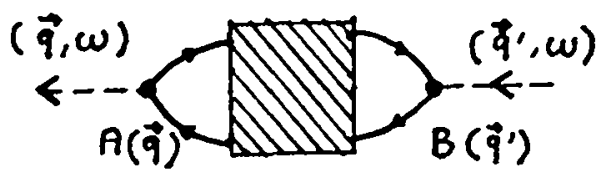

Figure 1 - The golarization propagator $\pi_{A B}\left(q, q^{\top} ; \omega\right)$
The response of the nucleus to any external excitation can be obtained from the polarization propagator $\pi_{A B}\left(\vec{q}, q^{\overrightarrow{1}} ; \omega\right)$ depicted schematically on fig. 9. Here the end point aperators $A(\vec{q})$ and $B(\vec{q})$ will be either the longitudinal spin-isospin operator $L(\vec{q})=\vec{\sigma} . \hat{q} \tau^{\alpha}$ or a component of the transverse spin-isospin operator $T_{(\vec{q}}(\vec{q})=$ $(\vec{\sigma} \times \hat{q}) \tau$. These polarization propăgators are considered as one body operators, written in momentum space, and are expressible in terms of their Wigner-transforms (WT) $\left[\pi_{A B}\right]_{W}(\vec{R}, \vec{K} ; \omega)$ according to :

$$
\prod_{A B}\left(\vec{k}+\frac{\vec{k}}{2}, \vec{k}-\frac{\vec{k}}{2} ; \omega\right)=\int d^{3} R e^{i \vec{k} \cdot \vec{R} / \vec{\hbar}}\left[\prod_{A B}\right]_{W}(\vec{k}, \vec{R} ; \omega)
$$

The semi-classical method consists in an expansion in power of $\hbar$ of the $W T\left[\pi_{A B}\right]_{W}$. In practice we will limit ourselves to order $\pi^{2}$. The response to an external weakly interacting probe, transferring to the nucleus a momentum-energy $\vec{q}, \omega$ and interacting with the nucleus through the one-body operator $A(\vec{q})$ is then obtained as :

$$
R_{A}(\vec{q} ; \omega)=-\frac{1}{\pi} \operatorname{Im}\left[\int d^{3} R\left[\Pi_{A A}\right]_{W}(\vec{q}, \vec{R} ; \omega)\right]
$$

\section{THE PARTICLE-HOLE (OR MEAN FIELD) RESPONSE FUNCTION}

We consider a spherical nucleus with $N=Z$ where the nucleons move in a central local single particle potential $V(R)$. The pure particle-hole response is independent of the spin-isospin structure of the excitation operators and writes very generally:

$$
\begin{aligned}
R_{0}(\vec{q} ; \omega)=\int d^{3} R \int \frac{d^{3} P}{\left(2 \pi \hbar^{3}\right)} \int \frac{d t}{2 \pi}\left[\theta\left(\epsilon_{F}-\hat{H}\right) e^{i \hat{H} t}\right]_{W}(\vec{P}, \vec{R}) \\
\times\left[\epsilon\left(\hat{H}-\epsilon_{F}\right) e^{-i \hat{H} t}\right]_{W}(\vec{p}+\vec{q}, \vec{R}) e^{i \omega t}
\end{aligned}
$$

where $\hat{H}$ est the single particle Hamiltonian and $\varepsilon_{F}$ is the Fermi energy. We expand the r.h.s. of Eq. (3) up to order $\hbar^{2}$. The familiar Thomas-Fermi result [1] is recovered as the first term (i.e. $\pi^{\circ}$ term) of the expansion. In the momentum range of interest, the surface-peaked $\pi^{2}$ contribution gives only a few per cent correction. We show on fig. 2 the result of the calculation for ${ }^{40} \mathrm{Ca}$ (Woods-Saxon potential with parameters taken from Myers [7] ) at momentum $q=350 \mathrm{MeV} / \mathrm{c}$. By comparison of the dotted curve (TF response) with the dashed curve $\left(\pi^{0}+\hbar^{2}\right.$ responses) it is seen that the $\pi^{2}$ contributions is positive at low energy $(\omega<100 \mathrm{MeV})$ and becomes negative at high energy. The smallness of the $\pi^{2}$ correction implicitely confirms the accuracy of TF theory, when we are dealing with average volume properties.

\section{THE RPA SPIN-ISOSPIN RESPONSES}

$\rightarrow \quad$ The response $R_{L}\left(R_{T}\right)$ to a longitudinal (transverse) spin-isospin excitation $\vec{\sigma} \cdot \hat{q} \tau^{\alpha}$ $\left(\vec{\sigma} \times \hat{q} \tau^{\alpha}\right)$ is calculated in the RPA ring approximation. The residual p.h interaction $V_{L}\left(V_{\gamma}\right)$ consists of pion (rho) exchange potential plus the short range $g^{\prime}$ interaction. The effect of 
antisymetrization is assumed to be incorporated in the Landau-Migdal parameters g'. This procedure has been justified by Stroth et al, at the TF level [2], i.e. antisymetrization yields a simple redefinition of $g$. The RPA polarization propagation $\pi$ is obtained as a solution of coupled integral equations which write in a schematic way :

$$
\begin{aligned}
& \pi_{L L}=\Pi_{L L}^{0}+\Pi_{L L}^{0} V_{L} \Pi_{L L}+\sum_{\mu} \Pi_{L T_{\mu}}^{0} V_{T} \Pi_{T_{\mu} L} \\
& \Pi_{T_{\mu} L}=\Pi_{T_{\mu} L}^{0}+\Pi_{T_{\mu L}}^{0} V_{L} \Pi_{L L}+\sum_{\nu} \Pi_{T_{\mu} T_{\nu}}^{0} V_{T} \Pi_{T_{\nu} L}
\end{aligned}
$$

Taking the Wigner-transform of Eq. 4, we get an equivalent set of equations which are solved order by order up to $\hbar^{2}$. For doing that, we need the WT of the mean field quantities. For instance $\left[\pi_{\mathrm{LI}}^{0}\right]_{w}$ has the form (omitting a tensorial piece irrelevant for spherical nuclei):

$$
\begin{aligned}
{\left[\pi_{L L}^{0}\right]_{W}(\vec{q}, \vec{R} ; \omega)=} & \alpha_{0}(\vec{q}, \vec{k} ; \infty)+\hbar^{2}\left\{\alpha_{2}(\vec{q}, \vec{R} ; \omega)\right. \\
& \left.+\left(1 / 3 q^{2}\right) \nabla_{R}^{2} \alpha_{0}(\vec{q}, \vec{R} ; \infty)\right\}+O\left(\hbar^{3}\right)
\end{aligned}
$$

Apart from the inclusion of $\Delta$-hole contribution which is specific to the spin-isospin channel, the first two terms (i.e. $\alpha$ and $\hbar^{2} \alpha_{2}$ ) of E.. 5 are independent of the particular form of the end point operators. Integrating their imaginary parts would give the response (3) calculated to order $\hbar^{2} . \alpha(\vec{q}, \vec{R} ; \omega)$ is formally identical to the polarization propagator of uncorrelated nuclear mattęr once one has introduced a local Fermi momentum $k_{F}(R)=$ $\left[2 M\left(\varepsilon_{F}-V(R)\right]^{1} /^{2}\right.$. The lengthy expression of $\alpha(\vec{q}, \vec{R} ; \omega)$ will be given in a forthcoming рарег. Note that the last term of Eq. (5) does not contribute to the $p$-h response. However it will become important in the renormalization procedure (see Eq. (10) below). From Eq. (4) we also see that there is a longitudinal transverse coupling term whose W-T writes :

$$
\left[\pi_{L T_{\mu}}^{0}\right]_{W}(\vec{q}, \vec{R} ; w)=-\left[\Pi_{T_{\mu}}^{c}\right]_{W}(\vec{q}, \vec{R} ; w)=\frac{1 \hbar}{q}\left(\hat{q} \times \vec{\nabla}_{R} \alpha_{c}(\vec{q}, \vec{R} ; w)\right)+O\left(\hat{\hbar}^{2}\right)
$$

One sees that the L-T mixing effect occurs only to order $\pi$ and thus disappears in the T-F approximation. After some algebra we find for the RPA longitudinal response :

$$
R_{L}(\vec{q} ; \omega)=-\frac{1}{\pi} \operatorname{Im}\left[\int d^{3} R\left\{\tilde{\alpha}_{O L}\left(\vec{q}, \vec{R}_{i} ; \omega\right)+\vec{h}^{2} \sum_{i=1}^{h} \tilde{\alpha}_{2 L}^{(i)}\left(\vec{q}_{i}, \vec{R}_{i} ;()\right)\right\}\right]
$$

Here $\tilde{\alpha}_{\mathrm{oL}}$ is the well known TF-RPA expression :

$$
\tilde{\alpha}_{O L}(\vec{q}, \vec{R} ; \omega)=\alpha_{0}(\vec{q}, \vec{R} ; \omega) \cdot /\left[1-V_{L}(\vec{q} ; \omega) \alpha_{0}(\vec{q}, \vec{R} ; \omega)\right]
$$

which yields renormalization and enhancement of the response. We have divided in Eq. (7) the $\hbar^{2}$ correction term in four distinct pleces. The first one may be understood as the renormalization of the $\hbar^{2}$ part of the mean-field response. It writes :

$$
\tilde{\alpha}_{2 L}^{(1)}=\alpha_{2} /\left[1-V_{L} \alpha_{0}\right]^{2}
$$

and also contributes to the enhancement of the response. However the most interesting (and the most important) $\hbar^{2}$ term is the second one :

$$
\tilde{\alpha}_{2 L}^{(2)}=\left(1 / 3 q^{2}\right)\left[\nabla_{R}^{2} \alpha_{0}+2 V_{T}\left(\vec{\nabla}_{R} \alpha_{0}\right)^{2} /\left(1-V_{T} \alpha_{0}\right)\right] /\left[1-V_{L} \alpha_{0}\right]^{2}
$$


which contains the $L T$ mixing term. It tends to attenuate the collective reshaping of the response. The last two $\hbar^{2}$ terms have the same effect but are less important for our cases of interest. Their expressions are quite complicated and involve gradients of $\alpha$ with respects to $\vec{q}$ and $\vec{R}$ as well as gradient of $V_{L}$ with respect to $\vec{q}$. As for the transverse response, $R_{T}$ is simply obtained by exchanging the role of $L$ and $T$ in Eqs. (7), (8) and (9). The effect of the $h^{2}$ terms is reversed i.e the quenching is reduced.

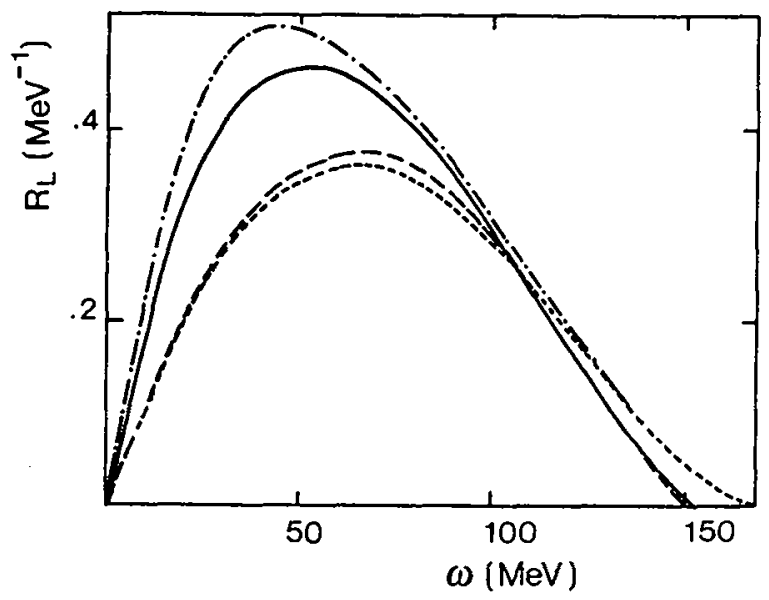

Figure 2 - Longitudinal spin-isospin responses for ${ }^{40} \mathrm{Ca}$ at $a=350$ MeU/c. The dotted line (mean-field responsel and the dot-dashed line (RPA response) correspond to a Thomas - Fermi calculation. The dashed line (mean-field response) and the full line (RPA response) are obtained after inclusion of the $\hbar^{2}$ corrections.

\section{RESULTS AND DISCUSSION}

For mathematical convenience the calculations have been carried out at finite temperature and the zero temperature case is obtained as a limit. In practice $T=1 \mathrm{MeV}$ is sufficient. On fig. 2, we show the results for the longitudinal response of ${ }^{40} \mathrm{Ca}$ at $q=350$ $\mathrm{MeV} / \mathrm{c}$. The Landau-Migdal parameter is taken to be $g^{\prime}=0.7$. At the TF level, we have a net collectivity as can be seen by comparison of mean field result (dotted line) with the RPA response (dot dashed line). When the $\pi^{2}$ corrections are included, the mean field response (dashed line) although slightly larger, remains very close to its TF analog but the RPA response (full line) is reduced, with respect to the TF-RPA one, by about ten per cent at low energy. Thus, once $\hbar^{2}$ corrections are added, the collectivity is a little reduced but still very net.

Unfortunately, the volume longitudinal response is not known experimentally. The only available information comes from the $\left(\vec{p}, p^{\vec{f}}\right)$ Los Alamos experiment [6] which essentially measures surface responses. Thus, the surface-peaked $\hbar^{2}$ term will obviously acquire a much more important relative weight yielding a considerable reduction of the collective effects. In addition the isoscalar spin responses also enter. In this experiment Carey and collaborators measured in fact the ratio.

$$
\tilde{x}=(1 / 2.3)\left(3.6 \tilde{R}_{L}(\sigma \sigma)+\tilde{R}_{L}(\sigma)\right) /\left(\tilde{R}_{T}(\sigma \tau)+R_{T}(\sigma)\right)
$$

at a momentum $q=350 \mathrm{MeV} / \mathrm{c}$. The tilde mean that we are dealing with surface responses and the distorsion effect is taken into account through a position dependent weight factor $C(R)$ multiplying the integrand in Eq. (7). $C(R)$ is derived in ref. [3]. In Eq. (11), we have approximated the isoscalar responses $R_{1}(\sigma, \tau)$ by the mean field one. The calculated ratio $\widetilde{X}$ for ${ }^{40} \mathrm{Ca}$ is displayed on Fig. 3. We see that the TF theory (dashed line) fails and the $\pi^{2}$ corrections considerably improve the agreement with data. The results for lead (not shown here) are very similar to those for Ca reflecting the fact that the surface properties do not depend very much on the mass number. Similar conclusions have been reached by Alberico et al. [8] within a (simplified) quantum mechanical RPA framework in a harmonic oscillator basis. Our method has the merit of flexibility, once the formalism is established, it is very 
easy with small computer time to perform calculation for different conditions, such as other incident energies or other probes "He-T. Although our results still remain above the data, they are quite conclusive and mainly show that the absence of contrast is mainly due to the peripheral nature of the probe. We should also mention that improvements in the theoretical description (such as inclusion of $2 p-2 h$ contribution, renormalization of the isoscalar responses [9]) is likely to further reduce the calculated ratio $\widetilde{x}$. From the above discussion, one can conclude that the access to the longitudinal volume response require further experiments. Charged pion electroduction $\left(e, e^{\prime} \pi^{2}\right)[10]$ could provide additional information in that direction but such an experiment is feasible only with the next generation of high duty cycle machines.

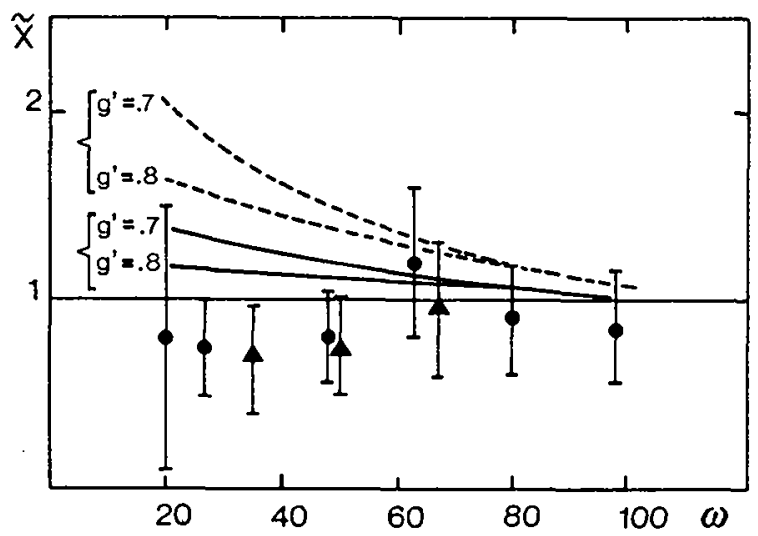

Figuze 3 - The ratio $\tilde{X}$ for ${ }^{40} \mathrm{Ca}$ as a function of the transferred energy $\omega$ at $q=350 \mathrm{MeV} / \mathrm{c}$. The dashed curves refer to a TF-RPA calculation and the full curves correspond to the RPA result including $\hbar^{2}$ corrections. The experimental data are from ref. [6].

\section{REFERENCES}

[1] STROTH, U., HASSE, R., SCHUCK, P., J. de Phys., C-6 (1984) 343

[2] STROTH, U. et al., Phys. Lett., 156-B (1985) 291

[3] STROTH, U., HASSE, R., SCHUCK, P., Nucl. Phys., A-462 (1987) 45

ALBERICO, W.M. et al., Nucl. Phys., A-462 (1987) 269

[4] MEZIANI, Z.E. et al., Phys. Rev. Lett., 54 (1985) 1233

[5] AlBeriCO, W.M., ERICSON, M., MOLINARI, A., Nucl. Phys., A-379 (1982) 429

[6] CAREY, T.A. et al., Phys. Rev. Lett., 53 (1984) 144

[7] MYERS, W.D., Nucl. Phys., A-145 (1970) 387

[8] ALBERICO, W.M. et al., Phys. Lett., 183-B (197) 135

[9] ORLANDINI, G., TRAINI, M., ERICSON, M., Phys. Lett., 179-B (1986) 201

[10] CHANFRAY, G., DELORME, J., Phys. Lett., 129-B (1983) 167

CHANFRAY, G., Nucl. Phys. A-429 (1984) 489 